\title{
Dual labeling with 5-aminolevulinic acid and fluorescein in high-grade glioma surgery with a prototype filter system built into a neurosurgical microscope: technical note
}

\author{
Eric Suero Molina, MD, MBA, Christian Ewelt, MD, Nils Warneke, MD, Michael Schwake, MD, \\ Michael Müther, MD, Stephanie Schipmann, MD, and Walter Stummer, MD \\ Department of Neurosurgery, University Hospital of Münster, Germany
}

OBJECTIVE Recent efforts to improve visualization of 5-aminolevulinic acid (5-ALA)-induced protoporphyrin IX (PPIX) fluorescence resulted in a dual-labeling technique, combining it with fluorescein sodium in a prototype setup. Fluorescein identifies regions with blood-brain barrier breakdown in gliomas. However, normally perfused and edematous brain fluoresces unselectively, with strong background enhancement. The aim of this study was to test the feasibility of a novel, integrated filter combination using porphyrins for selective tumor identification and fluorescein for background enhancement.

METHODS A microscope with a novel built-in filter system (YB 475) for visualizing both fluorescein and 5-ALA-induced porphyrins was used. Resection limits were identified with the conventional BLUE 400 filter system. Six patients harboring contrast ring-enhancing lesions were analyzed.

RESULTS The complete surgical field could now be illuminated. Fluorescein was helpful for improving background visualization, and enhancing dura, edematous tissue, and cortex. Overlapping regions with both fluorophores harbored merged orange fluorescence. PPIX fluorescence was better visualized, even in areas beyond a normal working distance of approximately $25 \mathrm{~cm}$, where the BLUE 400 filters recognized no or weak fluorescence.

CONCLUSIONS The novel filter system improved general tissue brightness and background visualization, enhancing fluorescence-guided tumor resection. Furthermore, it appears promising from a scientific perspective, enabling the simultaneous and direct observation of areas with blood-brain barrier breakdown and PPIX fluorescence.

https://thejns.org/doi/abs/10.3171/2018.12.JNS182422

KEYWORDS ALA; fluorescein; dual labeling; high-grade glioma; protoporphyrin IX; oncology; diagnostic technique

$\mathrm{M}$ ALIGNant gliomas carry a poor prognosis compared to other malignant tumors in humans, ${ }^{13,14}$ which is only slowly improving. ${ }^{11,36}$ It is generally agreed that the extent of resection and local tumor control are associated with a longer overall and progression-free survival, thus safe maximal resection is an important first step for multimodal therapy. ${ }^{12-14,17,21}$ Because even small remnants of tumor tissue can impair overall survival,, 28 surgical techniques and adjuncts are constantly being refined.

Because of the diffuse growth of malignant gliomas, it is difficult to identify tumor borders based on the microscope impression under white light or tissue consistency alone ${ }^{4}$ For this reason, several tools have emerged that enable better identification of marginal tumor. One method, 5-aminolevulinic acid (5-ALA)-induced protoporphyrin IX (PPIX) fluorescence-guided surgery (FGS), has proved to be particularly useful due to the accuracy of visualization and the fact that visualization occurs in real time, while operating. $8,29,30$ This compound is now approved by the European Medicines Agency and the US FDA. PPIX fluorescence tissue extends beyond the bor- 
ders of gadolinium contrast-enhancement in MRI, which for a long time has been the goal for maximal resection in malignant glioma surgery. ${ }^{38}$ Complementary tools, i.e., intraoperative ultrasonography, monitoring and mapping techniques, intraoperative imaging with CT or MRI, and neuronavigation, together with FGS, are the mainstays of modern glioma surgery. ${ }^{34}$ However, background illumination in FGS with 5-ALA is in some cases considered too weak, limiting background discrimination and requiring frequent changes between white light and blue light illumination, ${ }^{34}$ such as for hemostasis. Regarding this constraint, we see room for improvement. Fluorescein, a dye that is approved for ophthalmology, has been used for identifying areas of blood-brain barrier breakdown in the brain..$^{-3,16,23,34}$ However, this fluorophore, being in vessels and without specific affinity to tumor cells, also highlights perfused dura, normal brain, and areas of edema. When normal brain tissue is infringed by surgery, this leads to unspecific extravasation of fluorescein, requiring much experience to discriminate fluorescein from tumor-related blood-brain barrier disruption, surgical manipulation, or edema propagation ${ }^{7,23}$ (Fig. 1). On the other hand, 5-ALA PPIX-induced fluorescence bleaches rapidly and can be destroyed by white light or coagulation. We hypothesized that a combination of fluorescence information from both fluorophores might provide synergies during FGS, not only providing additional information during resection under fluorescence illumination, but also providing a platform for scientific evaluations. We recently published a proofof-principle study regarding a combination of the Pentero microscope YELLOW 560 module (Carl Zeiss Meditec) with a handheld device and external blue-light illumination (D-light C System, 375-440 nm, Storz). ${ }^{40}$ With the excitation maximum of fluorescein being $480 \mathrm{~nm}$ and of 5-ALA being $405 \mathrm{~nm}$, none of the existing filter systems to date was truly capable of simultaneously visualizing both fluorophores.

We now give the first report of the intraoperative use of a novel filter construct for detecting fluorescence (YB 475; Carl Zeiss Meditec) in combination with a tailored excitation light range, both incorporated into a standard surgical microscope. Detection and illumination filters were specifically engineered to allow simultaneous visualization of both fluorophores through a single optical pathway.

\section{Methods}

\section{Dual-Labeling Filter YB $\mathbf{4 7 5}$}

Fluorescein generates its strongest fluorescence at an excitation light wavelength of $480 \mathrm{~nm},,^{24,41}$ and its fluorescence maximum is at $525 \mathrm{~nm}$. Unlike fluorescein, the PPIX excitation maximum is found at $405 \mathrm{~nm},{ }^{15}$ delivering the highest fluorescence peak at $635 \mathrm{~nm}$ and a second peak at $704 \mathrm{~nm}^{15,19}$ (Fig. 2).

We used a modified experimental system with xenon light delivering an excitation wavelength in the range of 390-475 nm, after obtaining CE certification for medical usage (Carl Zeiss Meditec). The system was functionally fully integrated into a standard OPMI Pentero microscope, in addition to a BLUE 400 filter component for standard FGS. By allowing emission light to pass between 545 and

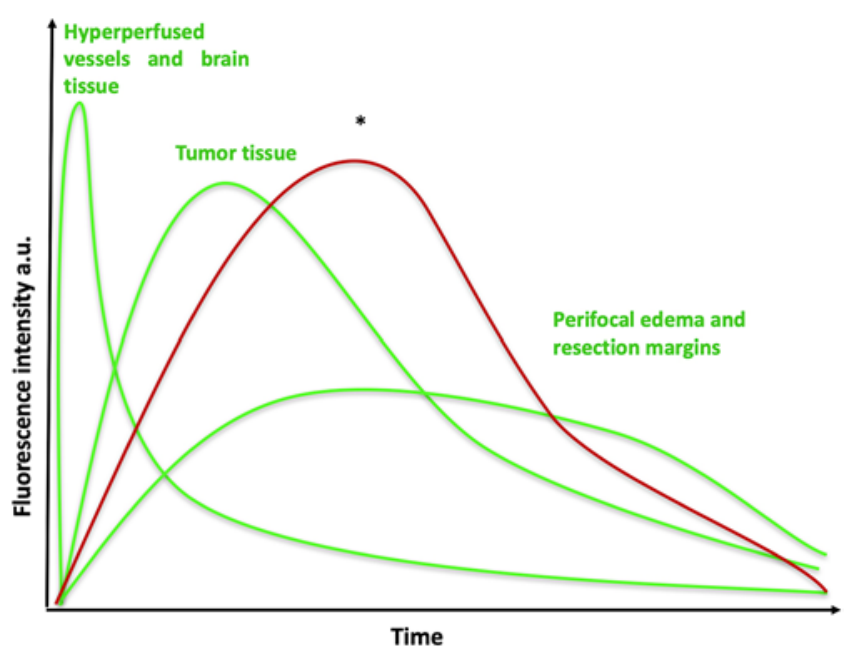

FIG. 1. Fluorescence time kinetic estimation curves for fluorescein (green) and PPIX (red) fluorescence. This scheme estimates the fluorescein kinetics as previously published. ${ }^{39}$ First, we observe fluorescein fluorescence in hyperperfused vessels and brain tissue. Hereafter, we observe what we call the "pseudoselectivity" for fluorescein in tumor tissue ${ }^{39,40}$ being finally observed in the peritumoral edema zone and zones of resection margins. In contrast, 5-ALA real-time kinetics (asterisk) was studied in a recent study from our group. We observed PPIX fluorescence to peak approximately 8 hours after administration (unpublished data). a.u. = arbitrary unit. Figure is available in color online only.

$740 \mathrm{~nm}$, the built-in long-pass filter allows visualization of both fluorophores PPIX and fluorescein (Fig. 2). In addition, the emission filter was equipped with an additive excitation bandpass component, allowing light passage between 430 and $455 \mathrm{~nm}$, thereby enabling partial passage of remitted blue excitation light for further enhancement of background tissue detail. Without fluorescein, the filter has similarities to the commercially available BLUE 400, however, the larger wavelength range of xenon excitation light allows better excitation of fluorescein fluorescence. For the purpose of this report, we have termed the prototype filter YB 475 (Fig. 2). During surgery, working distance was maintained at approximately $25 \mathrm{~cm}$. Light intensity was automatically upregulated to $100 \%$ when switching to surgery with BLUE 400 or YB 475 filter systems.

Due to technical constraints, the internal Zeiss Pentero HD1 video camera was not able to record pictures during surgery and video material was only obtainable for the new filter system YB 475 . Therefore, we provide screenshots from videos recorded with the YB 475 filter.

\section{Patient Collective}

Six patients harboring suspected malignant glioma were operated on at our institution between November 2016 and May 2017 and prospectively included in this study. 5-ALA, in a standard dose of $20 \mathrm{mg} / \mathrm{kg}$ body weight (Gliolan, Medac) was administered orally 4 hours prior to induction of anesthesia, and low-dose fluorescein (3 mg/ kg body weight; Fluorescein Alcon 10\%, Alcon Pharma $\mathrm{GmbH}$ )-which is still off-label for its application in neurosurgery-was administered intravenously during induction of anesthesia, as previously described. ${ }^{1,30}$ All surgeries were performed by senior neurosurgeons. 


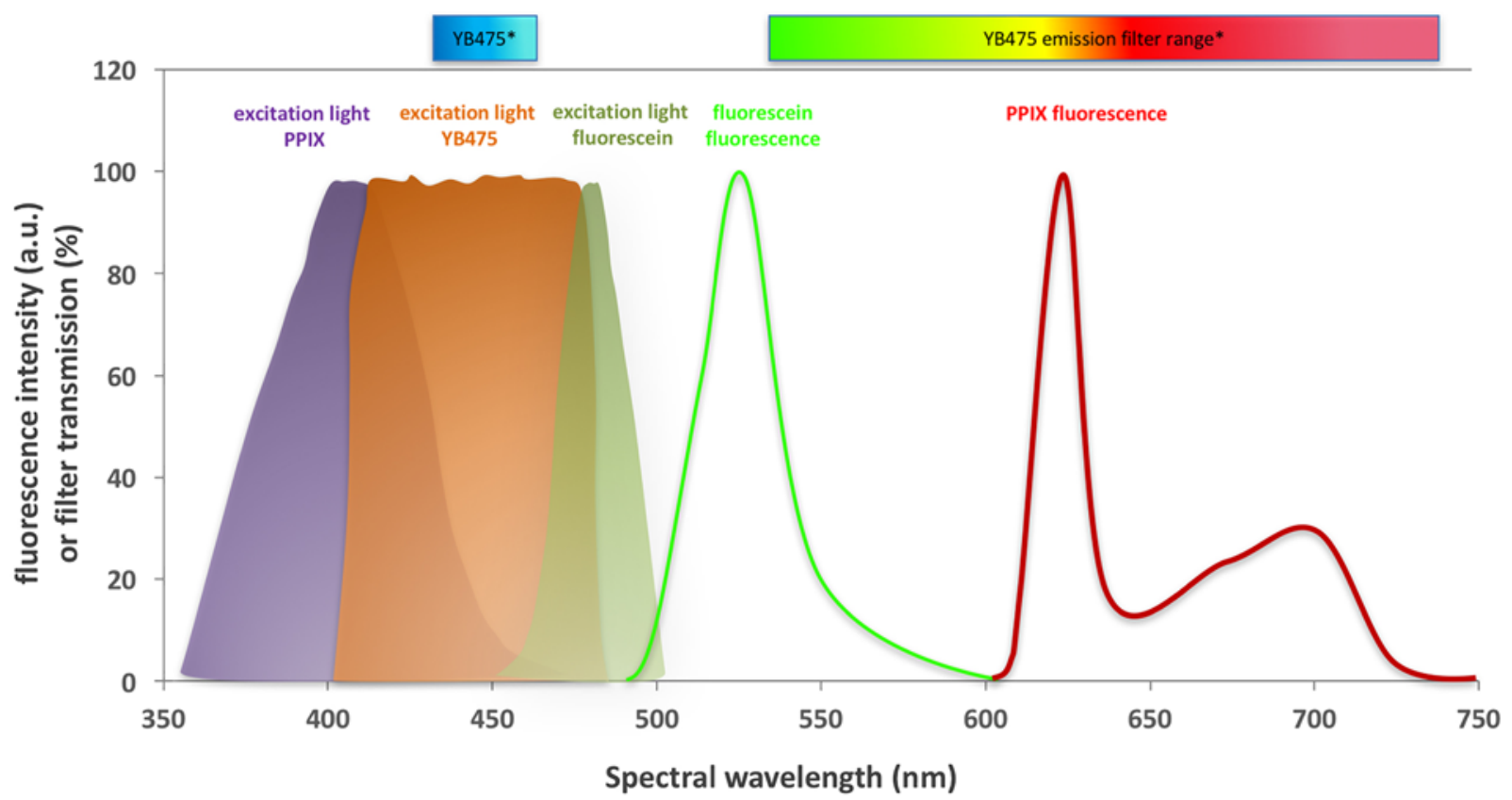

FIG. 2. Manufacturers' specifications of the new YB 475 filter system demonstrating an excitation light band that ranges from approximately 390 to $475 \mathrm{~nm}$, enabling light to be absorbed by PPIX and fluorescein simultaneously. The observation filter allows light to pass between 545 and $740 \mathrm{~nm}$. This setting enables optimal visualization for 5-ALA and fluorescein. In addition, the emission filter also allowed passage of a small band of light between 430 and $455 \mathrm{~nm}$, enabling remitted excitation light to pass and to further enhance background detail. a.u. = arbitrary unit. Figure is available in color online only.

We obtained written informed consent from all patients presented in this study after consultation with the ethics committee of the University of Münster concerning compassionate use of fluorescein, due to the off-label nature of this application. Each patient was discussed in an interdisciplinary setting within the tumor board from our institution, where surgery was proposed independently of this study. Mapping and monitoring included motor-evoked potentials by high frequency stimulation of motor structures in anesthetized patients, language mapping and monitoring under dexmedetomidine, ${ }^{37}$ neuronavigation, and ultrasonography.

No surgical decisions were made based on the images from the YB 475 filter. Because this was an observational study, no tissue was taken while solely applying the YB 475 filter. As standard protocol after glioma resection, early postoperative MRI was performed within 48 hours after surgery. In the T1-weighted MR images with and without gadolinium enhancement, complete resection of enhancing tumor was defined when residual tumor tissue of less than $0.175 \mathrm{~cm}^{3}$ or one voxel was detected., ${ }^{9,13,22,35}$

\section{Results}

Contrary to our previously described application, ${ }^{40}$ where only a limited part of the surgical field was illuminated for excitation of PPIX, the complete surgical area was now highlighted, maximizing the advantages of the dual-labeling technique.

Fluorescein again was observed in the dura, cortex, and edematous brain tissue, and its distribution differed from the PPIX tumor fluorescence. For example, central tumor necrosis showed weak fluorescein fluorescence but no PPIX accumulation. Furthermore, unspecific extravasation of fluorescein was noted during resection and at resection margins. From our observation, fluorescein did not obstruct PPIX fluorescence detection.

Even when using the BLUE 400 filter, fluorescein fluorescence was visible, especially in the normal cortex. However, using the new YB 475 filter construct, fluorescein fluorescence in tissue was strongly enhanced. In all studied cases, we observed red to orange PPIX fluorescence in tumor tissue, which indicates PPIX and fluorescein co-emitting red and yellow fluorescence (Fig. 3, Video 1). When directly comparing PPIX distributions under standard BLUE 400 and YB 475 filter moieties, we could not detect a difference in their borders (Video 1).

VIDEO 1. Intraoperative video of a 55-year-old woman harboring a glioblastoma in her right parietal lobe. The video demonstrates the visualization of fluorescent tumor tissue after receiving both 5-ALA and fluorescein as described in the paper with the BLUE 400 and the YB 475 dual-labeling filter. Note the richer background visualization utilizing the prototype filter. Copyright Eric Suero Molina. Published with permission. Click here to view.

Necrosis, when encountered, harbored a weak yellow fluorescence that served to highlight the cavity but also helped identify necrotic tissue. Blood in the resection cavity covered both fluorescence types. We considered this observation particularly useful because we could now interpret lack of any fluorescence due to covering with blood, rather than due to resection of all tumor (Video 2).

VIDEO 2. Intraoperatively acquired imaging of a 43-year-old man with a left-frontal distant recurrence of a gliosarcoma. Visualization 

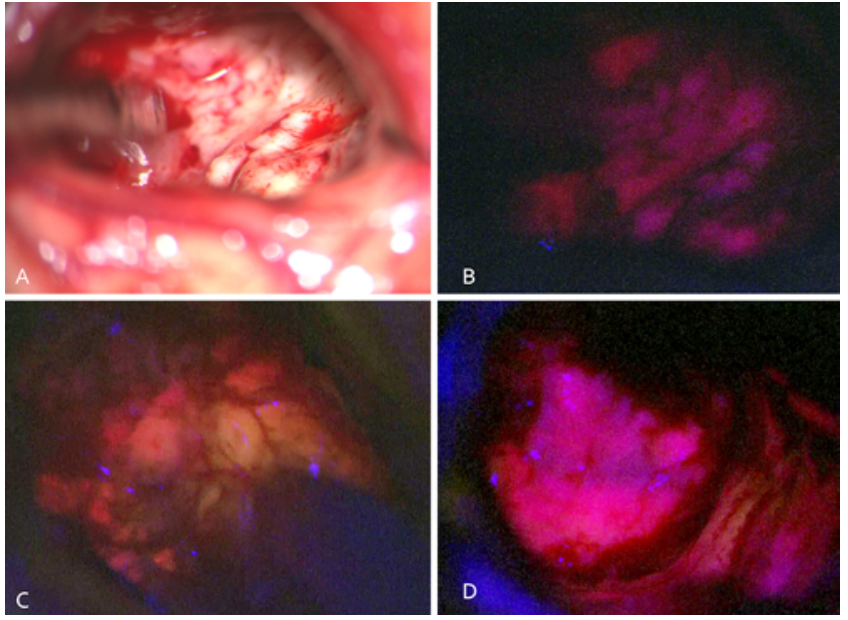

FIG. 3. Intraoperative imaging of a 72-year-old woman with a right postcentral glioblastoma using white-light microscopy (A), BLUE 400 (B), and YB 475 (C and D). Light intensity was $100 \%$ and working distance approximately $25 \mathrm{~cm}$. Panel $\mathrm{C}$ demonstrates a brighter background and a red/orange fluorescence. Panel $D$ shows a brighter background and the extent of fluorescence that is perceived with more background illumination. Figure is available in color online only.

is shown under both BLUE 400 and YB475 filter system. 5-ALAinduced fluorescence appeared red to orange. Fluoresceinfluorescence, additional to the tumor delineation due to PPIX fluorescence, enhances visualization of the surgical field, including tumor and adjacent tissue, and facilitates hemostasis. Copyright Eric Suero Molina. Published with permission.

The surrounding intense, yellow, fluorescein-fluorescence enhanced the surgical field, illuminating our surgical background and creating a brighter operative setting (Figs. 3 and 4). Additionally, deeply located structures beyond our area of focus in the surgical field were enhanced and much better visualized, facilitating orientation while operating. For hemostasis, changing between white-light microscopy and YB 475 was rarely required. Cortical vessels were easily distinguished on the yellow background of perfused tissue and localized sources of bleeding during resection could be much better observed under the YB 475 filter compared to the BLUE 400 and consequently coagulated during surgery. No adverse effects were observed either from 5-ALA or fluorescein in these patients.

\section{Discussion}

FGS with 5-ALA has become a highly recognized standard for enhancing resection of high-grade gliomas. As a natural heme precursor, 5-ALA induces the expression of fluorescent PPIX in tumor cells..$^{32}$ Due to the intracellular accumulation of porphyrins, there are no issues of unspecific leakage of fluorophore or propagation with edema using this method. The excitation maximum of 5-ALA-derived porphyrins is $405 \mathrm{~nm}$, representing the Soret band of PPIX, ${ }^{33}$ inducing an emission peak at 635 $\mathrm{nm}$ together with a secondary peak at $704 \mathrm{~nm} \cdot{ }^{15,19}$ The present filter system BLUE 400 (Carl Zeiss Meditec) has been left unchanged since its initial introduction in the late $1990 \mathrm{~s}^{29}$ and innovations should be considered.
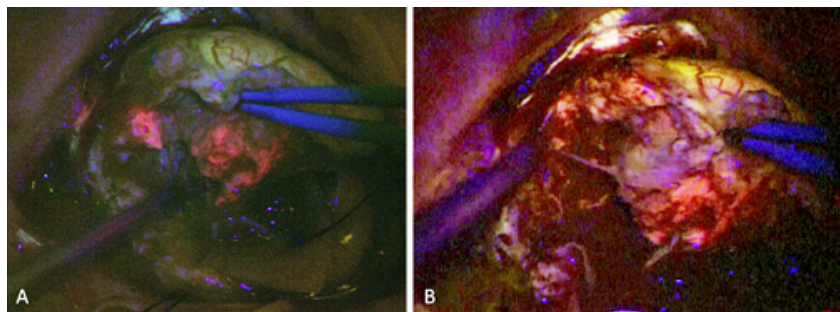

FIG. 4. Images obtained in a 43-year-old man with a left-frontal distant recurrence of a gliosarcoma. Intraoperative imaging was performed with a BLUE 400 (A) and YB 475 filter (B) after 5-ALA and fluorescein application as described in the Methods. The synergistic effects of fluorescein with 5-ALA could partly be appreciated using the standard BLUE 400 filter but was markedly enhanced using the filter moiety. Note the brighter background and the merged red-orange fluorescence observed during the dual-labeling technique with the YB 475 filter. Furthermore, observe yellow illumination of the cortex with negatively contrasted vessels, which were thus easily identified. Figure is available in color online only.

\section{Fluorescein in Malignant Glioma Surgery}

When used as a singular agent for depicting tumor via the impaired blood-brain barrier, fluorescein has a number of possible pitfalls, which pose potential risks. ${ }^{23,25,40}$ Timing is a crucial factor when working with fluorescein. Initially and promptly after intravenous application, fluorescein will have high concentration in plasma and thus in vessels, and all perfused tissues, thus in the brain. ${ }^{20}$ Subsequently, fluorescein enters tumor extracellular space in areas in which the blood-brain barrier is disrupted, from there spreading with edema into peritumoral tissue. ${ }^{39}$ In humans, the rate of tumor edema propagation is about 2 $\mathrm{mm} / \mathrm{hr} .^{10,27,39}$ The period of time between extravasation and significant incursion into peritumoral tissue offers a window of "pseudoselectivity" in which this agent will provide information about the angiogenic part of the tumor, ${ }^{40}$ thereafter additionally marking perifocal edema tissue. ${ }^{23,27,39,40}$ When brain tissue is manipulated, i.e., during corticotomy, fluorescein leaks from the brain tissue interface unselectively. Fluorescein has no known affinity to malignant glioma cells. ${ }^{6}$

However, the disadvantages of fluorescein outlined above might be used to its advantage when combining both fluorophores, making fluorescein the perfect adjunct for a dual-labeling technique with 5-ALA.

\section{Advantages of Dual-Labeling and the YB 475 Filter Construct}

In our experience, we observed the background to be more easily distinguishable due to the yellow fluorescence emitted from intravascular fluorescein in perfused brain. Furthermore, blood in vessels, which is dark, nicely contrasts cortical and subcortical vessels against yellowstained brain tissues, thus allowing tissue manipulation or vessel coagulation under the fluorescence mode of the microscope.

The advantages of this better background illumination are the most important observation from this technique assessment. Neurosurgeons accustomed to 5-ALA-derived fluorescence-guided surgery often desire a better 
intraoperative background image and excellent delineation of cortical vessels. This technique assessment demonstrates that a filter combination could be devised that can be integrated into a standard neurosurgical microscope.

Even though the optical images acquired through the microscopes, as visually perceived, are of high quality, we previously demonstrated that the quality of the intraoperatively acquired images differs immensely compared to what is perceived by the human eye while looking through the surgeon's ocular. ${ }^{40}$ Therefore, available imaging and video material in this paper is limited to the existing documentation and is unfortunately poor compared to the optical perception.

\section{Outlooks}

There is a strong time dependency of fluorescein-derived background enhancement. Fluorescein is eliminated with a half-life of 264 minutes. ${ }^{26}$ In our first assessment, we chose to apply fluorescein as previously described, i.e., $3 \mathrm{mg} / \mathrm{kg}$ body weight administered during induction of anesthesia. ${ }^{1}$ Because applying fluorescein does not have issues related to tumor selectivity, when used as a background marker, different doses administered at different time points or re-administration (such as when concentrations become too low) should also be considered. Care must be taken to keep fluorescein concentrations low, however. We have found higher $\operatorname{doses}^{23}$ or a later application ${ }^{25}$ to greatly confound image interpretation due to high intravascular and thus intraparenchymal fluorescein concentrations. Furthermore, leakage of fluorescein with blood and contamination of the brain and the surgical cavity could also contribute to this. ${ }^{23}$ For this reason, we have adapted the protocol suggested by Acerbi et al., ${ }^{1,3}$ administering low doses of fluorescein with induction of anesthesia. In contrast, PPIX fluorescence, which is synthesized within tumor cells, reaches a maximum concentration 6-8 hours after oral administration and maintains a strong contrast up to 12 hours after 5-ALA administration. ${ }^{31}$

Finally, using both fluorophores will provide information on tumor cells and their capacity to accumulate PPIX, as well as on blood-brain barrier integrity, edema distribution, and propagation from fluorescein fluorescence. Thus, using these technologies in conjunction will effectively allow intraoperative discrimination of necrosis, solidly proliferating tumor, and infiltrating tumor down to a tumor cell density of about $10 \%, 35$ as well as the zone of peritumoral edema. Furthermore, reducing the need for white light illumination during resection will minimize the risk for fluorophores to bleach, which is one of the limitations of 5-ALA. The technique thus provides a platform for interesting scientific questions involving selective biopsies from these areas, as previously described for 5-ALA-induced porphyrins for discriminating infiltrating tumor. ${ }^{18}$

A randomized study directly comparing surgical times using either method (BLUE 400 vs YB475) is presently in the planning phase in which operating times under fluorescence and white light as well as the overall duration of surgery will be measured.

\section{Limitations}

Studies for unequivocally determining advantages of the dual-labeling technique are pending and are constructed around surrogate indicators of a benefit, e.g., the duration of surgery and possibly completeness of resection; however, we do not believe it necessary to demonstrate advantages in terms of survival. Because this is a tool, any results of resection will depend more strongly on the surgeon and his usage of an adjunct, rather than on the adjunct itself.

Moreover, fluorescein is still off-label in the setting of malignant glioma surgery. Hence, at least in Europe, it is difficult to apply this compound in a larger context. Furthermore, this was an observational study and no histological examination of acquired tissue under the new filter system was conducted. The quality of images was restricted to available video technology, which is greatly inferior to the optical perception and poses challenges for demonstrating versatility in a publication. Notwithstanding, we believe this to have demonstrated the principle perception that a new visualization tool for PPIX fluorescence could enhance our surgical experience in malignant glioma surgery.

\section{Conclusions}

The dual-labeling technique with a new filter moiety, the YB 475 filter construct, appears to be a promising approach to improve visualization for FGS with ALA. Under fluorescence light, the cortex and vessels were well visualized, and discrimination of deep structures was enhanced. Effort should now be made to approve fluorescein for neurosurgery as a marker of blood-brain barrier breakdown. Its off-label status still impairs its general use in neurosurgery, with separate informed consent being necessary with every single use.

\section{References}

1. Acerbi F, Broggi M, Broggi G, Ferroli P: What is the best timing for fluorescein injection during surgical removal of high-grade gliomas? Acta Neurochir (Wien) 157:13771378,2015

2. Acerbi F, Broggi M, Eoli M, Anghileri E, Cavallo C, Boffano $\mathrm{C}$, et al: Is fluorescein-guided technique able to help in resection of high-grade gliomas? Neurosurg Focus 36(2): E5, 2014

3. Acerbi F, Broggi M, Eoli M, Anghileri E, Cuppini L, Pollo B, et al: Fluorescein-guided surgery for grade IV gliomas with a dedicated filter on the surgical microscope: preliminary results in 12 cases. Acta Neurochir (Wien) 155:1277-1286, 2013

4. Albert FK, Forsting M, Sartor K, Adams HP, Kunze S: Early postoperative magnetic resonance imaging after resection of malignant glioma: objective evaluation of residual tumor and its influence on regrowth and prognosis. Neurosurgery 34:45-61, 1994

5. Coburger J, Hagel V, Wirtz CR, König R: Surgery for glioblastoma: impact of the combined use of 5-aminolevulinic acid and intraoperative MRI on extent of resection and survival. PLoS One 10:e0131872, 2015

6. Diaz RJ, Dios RR, Hattab EM, Burrell K, Rakopoulos P, Sabha N, et al: Study of the biodistribution of fluorescein in glioma-infiltrated mouse brain and histopathological correlation of intraoperative findings in high-grade gliomas re- 
sected under fluorescein fluorescence guidance. J Neurosurg 122:1360-1369, 2015

7. Diez Valle R, Tejada Solis S: Answer to: "sodium fluorescein-guided resection under the YELLOW 560-nm surgical microscope filter in malignant brain tumor surgery-a feasibility study" (April 2013, volume 155, issue 4, pp 693-69). Acta Neurochir (Wien) 155:1319-1320, 2013

8. Eljamel S: 5-ALA fluorescence image guided resection of glioblastoma multiforme: a meta-analysis of the literature. Int J Mol Sci 16:10443-10456, 2015

9. Floeth FW, Stummer W: The value of metabolic imaging in diagnosis and resection of cerebral gliomas. Nat Clin Pract Neurol 1:62-63, 2005

10. Gröger U, Huber P, Reulen HJ: Formation and resolution of human peritumoral brain edema. Acta Neurochir Suppl (Wien) 60:373-374, 1994

11. Johnson DR, Omuro AMP, Ravelo A, Sommer N, Guerin A, Ionescu-Ittu R, et al: Overall survival in patients with glioblastoma before and after bevacizumab approval. Curr Med Res Opin 34:813-820, 2018

12. Kreth FW, Thon N, Simon M, Westphal M, Schackert G, Nikkhah G, et al: Gross total but not incomplete resection of glioblastoma prolongs survival in the era of radiochemotherapy. Ann Oncol 24:3117-3123, 2013

13. Lacroix M, Abi-Said D, Fourney DR, Gokaslan ZL, Shi W, DeMonte F, et al: A multivariate analysis of 416 patients with glioblastoma multiforme: prognosis, extent of resection, and survival. J Neurosurg 95:190-198, 2001

14. McGirt MJ, Chaichana KL, Gathinji M, Attenello FJ, Than $\mathrm{K}$, Olivi A, et al: Independent association of extent of resection with survival in patients with malignant brain astrocytoma. J Neurosurg 110:156-162, 2009

15. Montcel B, Mahieu-Williame L, Armoiry X, Meyronet D, Guyotat J: Two-peaked 5-ALA-induced PpIX fluorescence emission spectrum distinguishes glioblastomas from low grade gliomas and infiltrative component of glioblastomas. Biomed Opt Express 4:548-558, 2013

16. Neira JA, Ung TH, Sims JS, Malone HR, Chow DS, Samanamud JL, et al: Aggressive resection at the infiltrative margins of glioblastoma facilitated by intraoperative fluorescein guidance. J Neurosurg 127:111-122, 2017

17. Orringer D, Lau D, Khatri S, Zamora-Berridi GJ, Zhang K, Wu C, et al: Extent of resection in patients with glioblastoma: limiting factors, perception of resectability, and effect on survival. J Neurosurg 117:851-859, 2012

18. Piccirillo SG, Dietz S, Madhu B, Griffiths J, Price SJ, Collins VP, et al: Fluorescence-guided surgical sampling of glioblastoma identifies phenotypically distinct tumour-initiating cell populations in the tumour mass and margin. Br J Cancer 107:462-468, 2012

19. Pogue BW, Gibbs-Strauss S, Valdés PA, Samkoe K, Roberts DW, Paulsen KD: Review of neurosurgical fluorescence imaging methodologies. IEEE J Sel Top Quantum Electron 16: $493-505,2010$

20. Roberts DW, Olson J: Fluorescein guidance in glioblastoma resection. N Engl J Med 376:e36, 2017

21. Sanai N, Berger MS: Extent of resection influences outcomes for patients with gliomas. Rev Neurol (Paris) 167:648-654, 2011

22. Schucht P, Beck J, Abu-Isa J, Andereggen L, Murek M, Seidel $\mathrm{K}$, et al: Gross total resection rates in contemporary glioblastoma surgery: results of an institutional protocol combining 5-aminolevulinic acid intraoperative fluorescence imaging and brain mapping. Neurosurgery 71:927-936, 2012

23. Schwake M, Stummer W, Suero Molina EJ, Wölfer J: Simultaneous fluorescein sodium and 5-ALA in fluorescence-guided glioma surgery. Acta Neurochir (Wien) 157:877-879, 2015

24. Sjoback R, Nygren J, Kubista M: Absorption and fluores- cence properties of fluorescein. Spectrochim Acta A Mol Biomol Spectrose 51:L7-L21, 1995

25. Stummer W: Factors confounding fluorescein-guided malignant glioma resections: edema bulk flow, dose, timing, and now: imaging hardware? Acta Neurochir (Wien) 158:327328,2016

26. Stummer W: Poor man's fluorescence? Acta Neurochir (Wien) 157:1379-1381, 2015

27. Stummer W, Götz C, Hassan A, Heimann A, Kempski O: Kinetics of Photofrin II in perifocal brain edema. Neurosurgery 33:1075-1082, 1993

28. Stummer W, Meinel T, Ewelt C, Martus P, Jakobs O, Felsberg J, et al: Prospective cohort study of radiotherapy with concomitant and adjuvant temozolomide chemotherapy for glioblastoma patients with no or minimal residual enhancing tumor load after surgery. J Neurooncol 108:89-97, 2012

29. Stummer W, Novotny A, Stepp H, Goetz C, Bise K, Reulen HJ: Fluorescence-guided resection of glioblastoma multiforme by using 5-aminolevulinic acid-induced porphyrins: a prospective study in 52 consecutive patients. J Neurosurg 93:1003-1013, 2000

30. Stummer W, Pichlmeier U, Meinel T, Wiestler OD, Zanella F, Reulen HJ: Fluorescence-guided surgery with 5-aminolevulinic acid for resection of malignant glioma: a randomised controlled multicentre phase III trial. Lancet Oncol 7:392401, 2006

31. Stummer W, Stepp H, Wiestler OD, Pichlmeier U: Randomized, prospective double-blinded study comparing 3 different doses of 5-aminolevulinic acid for fluorescence-guided resections of malignant gliomas. Neurosurgery 81:230-239, 2017

32. Stummer W, Stocker S, Novotny A, Heimann A, Sauer O, Kempski O, et al: In vitro and in vivo porphyrin accumulation by C6 glioma cells after exposure to 5-aminolevulinic acid. J Photochem Photobiol B 45:160-169, 1998

33. Stummer W, Stocker S, Wagner S, Stepp H, Fritsch C, Goetz $\mathrm{C}$, et al: Intraoperative detection of malignant gliomas by 5 -aminolevulinic acid-induced porphyrin fluorescence. Neurosurgery 42:518-526, 1998

34. Stummer W, Suero Molina E: Fluorescence imaging/agents in tumor resection. Neurosurg Clin N Am 28:569-583, 2017

35. Stummer W, Tonn JC, Goetz C, Ullrich W, Stepp H, Bink A, et al: 5-Aminolevulinic acid-derived tumor fluorescence: the diagnostic accuracy of visible fluorescence qualities as corroborated by spectrometry and histology and postoperative imaging. Neurosurgery 74:310-320, 2014

36. Stupp R, Mason WP, van den Bent MJ, Weller M, Fisher B, Taphoorn MJ, et al: Radiotherapy plus concomitant and adjuvant temozolomide for glioblastoma. N Engl J Med 352:987-996, 2005

37. Suero Molina E, Schipmann S, Mueller I, Wölfer J, Ewelt C, Maas M, et al: Conscious sedation with dexmedetomidine compared with asleep-awake-asleep craniotomies in glioma surgery: an analysis of 180 patients. J Neurosurg 129:12231230,2018

38. Suero Molina E, Schipmann S, Stummer W: Maximizing safe resections: the roles of 5-aminolevulinic acid and intraoperative MR imaging in glioma surgery-review of the literature. Neurosurg Rev [epub ahead of print], 2017

39. Suero Molina E, Stummer W: Where and when to cut? Fluorescein guidance for brain stem and spinal cord tumor surgery-technical note. Oper Neurosurg (Hagerstown) 15:325-331, 2018

40. Suero Molina E, Wölfer J, Ewelt C, Ehrhardt A, Brokinkel B, Stummer W: Dual-labeling with 5-aminolevulinic acid and fluorescein for fluorescence-guided resection of high-grade gliomas: technical note. J Neurosurg 128:399-405, 2018

41. Zhang XF, Zhang J, Liu L: Fluorescence properties of twenty fluorescein derivatives: lifetime, quantum yield, absorption and emission spectra. J Fluoresc 24:819-826, 2014 


\section{Disclosures}

Walter Stummer received speaker and consultant fees from Medac, Zeiss, Leica, Photonamic, and NXDC.

\section{Author Contributions}

Conception and design: Suero Molina, Stummer. Acquisition of data: Suero Molina, Ewelt, Warneke, Schwake, Muether, Stummer. Analysis and interpretation of data: Suero Molina, Stummer. Drafting the article: Suero Molina. Critically revising the article: Suero Molina, Ewelt, Warneke, Schipmann, Stummer. Reviewed submitted version of manuscript: all authors. Approved the final version of the manuscript on behalf of all authors: Suero Molina. Administrative/technical/material support: Suero Molina, Schipmann, Stummer. Study supervision: Stummer.

\section{Supplemental Information \\ Videos}

Video 1. https://vimeo.com/317270000.

Video 2. https://vimeo.com/317270031.

\section{Correspondence}

Eric Suero Molina: University Hospital of Münster, Germany. eric.suero@ukmuenster.de. 